\title{
All forms of energy are equal, but some forms of energy are more equal than others
}

\author{
Santanu Bandyopadhyay ${ }^{1}$
}

Accepted: 27 October 2021 / Published online: 2 November 2021

(c) The Author(s), under exclusive licence to Springer-Verlag GmbH Germany, part of Springer Nature 2021

William Thomson (Lord Kelvin) combined two Greek words, thermotis (heat) and dynamis (power), to create the word thermodynamics. It was commenced as a subject to study the interrelations between heat and work. Over the years, it evolved to encompass the entire domain of energy conversion. The first law of thermodynamics asserts that different forms of energy are equivalent, and an appropriate conservation equation can be written. The first law analysis of a system aims to identify energy losses and, thereby, helps in improving the overall energy efficiency through energy conservation.

The first law analysis of an energy conversion process fails to identify the inherent losses and, therefore, cannot guarantee improvements in energy conversion. There are energy losses whenever one form of energy is transformed into another. In certain cases, the inherent losses during energy conversion are significant. Based on these inherent losses, different forms of energy are classified into two groups: high-quality energy (such as work and electricity) and low-quality energy (such as heat and wind). High-quality energy can be transformed into another high- or lowquality energy with extremely high conversion efficiency. On the other hands, low-quality energy cannot be converted to high-quality energy completely, not even theoretically. These limitations on energy conversion efficiencies are the consequence of the second law of thermodynamics.

Let us consider some examples of energy transformations. Carnot's efficiency plays an important role whenever heat (low-quality energy) is converted to work (high-quality energy). The second law of thermodynamics states that a heat engine that converts heat to work cannot be $100 \%$ efficient, and there has to be some heat rejection (Kelvin-Plank

Santanu Bandyopadhyay

santanub@iitb.ac.in

1 Department of Energy Science and Engineering, Indian Institute of Technology Bombay, Powai, Mumbai 400076, India statement of the second law of thermodynamics). The maximum efficiency of a heat engine is known as the Carnot efficiency. Whenever wind energy is converted to work in a wind turbine, the Betz limit steers the maximum work that can be produced. Similarly, the Shockley-Queisser limit plays a vital role in electricity production from solar radiation through a photovoltaic device. To determine the quality of any energy form, we should focus on the basic elements of the energy carrier. For high-quality energy, basic constituents carry the same amount of energy in the same direction. Otherwise, the form of energy is of low quality. Thermal energy is a manifestation of the internal motions of particles. Every particle moves with different velocities in different directions, and only their net effect manifests heat. Therefore, heat is low-quality energy. Various air molecules move with different velocities, and their net kinetic energy reflects the energy content in the wind. Wind energy is also low-quality energy. With an identical charge, every electron moves in the same direction to represent the higher quality of electricity. The second law of thermodynamics states that all forms of energy are not equivalent. There are some inherent losses whenever low-quality energy is converted to high-quality form. The second law analysis of a system accounts for these inherent and unavoidable energy losses and aims to identify avoidable energy conversion losses. By reducing these avoidable losses, the overall energy efficiency of a system can be achieved.

Identifications of different qualities of energy and losses during energy conversions are the critical steps in achieving sustainability. To deliver any energy service satisfactorily, primary energy has to undergo multiple transformations, and a significant conversion loss is encountered. For example, 94-96\% of the primary energy is lost to produce illuminations in modern electric lamps. Appropriate use of daylighting and incorporations of efficient electric lamps will lead to significant energy savings. Similarly, the need for thermal energy (for process or district heating) can be met more efficiently with solar thermal technologies than with 
solar photovoltaics by avoiding intermediate energy conversion stages. The subtle distinctions of different qualities of energy are going to play an essential role in achieving overall sustainability in the near future.
Publisher's Note Springer Nature remains neutral with regard to jurisdictional claims in published maps and institutional affiliations. 